\title{
Model-based spectral analysis of photon propagation through nanoparticle-labeled epithelial tissues
}

Can Cihan

Dizem Arifler 


\title{
Model-based spectral analysis of photon propagation through nanoparticle-labeled epithelial tissues
}

\author{
Can Cihan ${ }^{\mathrm{a}}$, Dizem Arifler ${ }^{* \mathrm{~b}}$ \\ ${ }^{\mathrm{a}}$ Department of Electrical and Electronics Engineering, Bilkent University, TR-06800 Bilkent, \\ Ankara, Turkey; \\ ${ }^{\mathrm{b}}$ Department of Physics, Eastern Mediterranean University, Famagusta, Cyprus
}

\begin{abstract}
Metal nanoparticles can function as optical contrast enhancers for reflectance-based diagnosis of epithelial precancer. We carry out Monte Carlo simulations to model photon propagation through normal tissues, unlabeled precancerous tissues, and precancerous tissues labeled with gold nanospheres and we compute the spectral reflectance response of these different tissue states. The results indicate that nanoparticle-induced changes in the spectral reflectance profile of tissues depend not only on the properties of these particles but also on the source-detector geometry used. When the source and detector fibers are oriented side by side and perpendicular to the tissue surface, the reflectance intensity of precancerous tissue is lower compared to that of normal tissue over the entire wavelength range simulated and addition of nanospheres enhances this negative contrast. When the fibers are tilted toward each other, the reflectance intensity of precancerous tissue is higher compared to that of normal tissue and labeling with nanospheres causes a significant enhancement of this positive contrast. The results also suggest that model-based spectral analysis of photon propagation through nanoparticle-labeled tissues provides a useful framework to quantify the extent of achievable contrast enhancement due to external labeling and to assess the diagnostic potential of nanoparticle-enhanced optical measurements.
\end{abstract}

Keywords: Epithelial precancer, Monte Carlo modeling, nanoparticles, optical spectroscopy, photon propagation, reflectance

\section{INTRODUCTION}

Numerous experimental and clinical studies have been carried out to assess the potential of optical spectroscopy and imaging in providing accurate diagnosis of epithelial precancer. These studies present sound evidence that optical signals are sensitive to morphological, structural and biochemical changes associated with cancer progression. ${ }^{1}$ There is currently a significant interest to develop optically active, molecular-specific labeling agents that can selectively bind to cancer biomarkers in tissues and enhance intrinsic optical signals. Metal nanoparticles strongly absorb and scatter light with distinct spectral features and they have been observed to function as contrast enhancers for reflectance-based diagnostics. ${ }^{2-4}$

The goal of the research presented in this work is to model photon propagation through normal tissues, unlabeled precancerous tissues, and precancerous tissues labeled with gold nanospheres. We perform Monte Carlo simulations to analyze the reflectance response of these different tissue states and we quantify the extent of signal and contrast enhancement due to external labeling. Since probing depth is strongly dependent on the optical sensor geometry employed, we simulate two fiber optic probe designs that demonstrate different levels of sensitivity to the top epithelial layer for which specific labeling can be achieved. The results obtained are expected to enable a comparative assessment of the effect of source-detector geometry on the observed contrast profile.

*dizem.arifler@emu.edu.tr; phone +90 392 630-1060; fax +90 392 365-1604.

Clinical and Biomedical Spectroscopy and Imaging II, edited by Nirmala Ramanujam, Jürgen Popp, Proc. of SPIE-OSA Biomedical Optics, SPIE Vol. 8087, 80872I · C 2011 SPIE-OSA · CCC code: 1605-7422/11/\$18 - doi: 10.1117/12.889421 


\section{METHODS}

Monte Carlo (MC) modeling provides a flexible approach to study photon propagation in multi-layered turbid media and it can also be adapted to quantify the overall optical response of tissues labeled with metal nanoparticles. ${ }^{5,6}$ The MC algorithm employed in this work was implemented in $\mathrm{C} / \mathrm{C}++$ and has been previously described. ${ }^{7-9}$

Epithelial tissue was modeled as consisting of two layers. The top epithelial layer was assigned a thickness of $300 \mu \mathrm{m}$ and the stromal layer underneath was assumed to be of infinite extent. The refractive indices of these two tissue layers were both set to 1.35 . The scattering and absorption coefficients and the anisotropy factors used to construct normal and unlabeled precancerous tissue were based on values reported in the literature. ${ }^{8}$ The angular scattering distributions in the epithelial and stromal layers were approximated with Henyey-Greenstein phase functions. When precancerous tissue is labeled with gold nanospheres targeted for cellular biomarkers, stromal optical properties remain unchanged, but the scattering and absorption properties of the epithelial layer need to be modified to account for the additional optical effect of these particles. ${ }^{5,6,10}$ Mie theory was used to compute the scattering and absorption cross sections and scattering phase functions of $80-\mathrm{nm}$ gold nanospheres; these results were then incorporated to MC modeling to analyze photon propagation with optical properties modified in accordance with a nanoparticle concentration of about $2 \times 10^{11}$ particles $/ \mathrm{mL}$, which is equivalent to a volume fraction of $0.005 \%$.

The source-detector geometries consisted of a single source fiber and a single detector fiber, both in contact with the tissue surface. Each fiber was assigned a $100-\mu \mathrm{m}$ diameter and a numerical aperture of 0.11 (in air). The refractive indices of the fibers were set to 1.5. The first geometry tested involved fibers that were perpendicular to the tissue surface and were separated by a center-to-center distance of $150 \mu \mathrm{m}$. In the second geometry, the distal ends of the fibers were tilted toward each other; each fiber made an angle of $30^{\circ}$ with respect to the tissue normal, but the center-to-center separation was again kept at $150 \mu \mathrm{m}$.

Simulations were carried out with $10^{8}$ photons for every $20 \mathrm{~nm}$ over the wavelength range of 400 to $700 \mathrm{~nm}$. Each simulation was repeated three times and the results presented represent averages over three simulations. Intensities at these sixteen wavelengths were then interpolated to generate spectral plots with increments of $2.5 \mathrm{~nm}$.

\section{RESULTS}

\subsection{Optical properties}

Figure 1 shows the epithelial scattering and absorption coefficients used in the simulations. We assume that precancer progression does not change the absorption properties of the epithelium and hence the absorption coefficients for normal and unlabeled precancerous tissue have the same values.

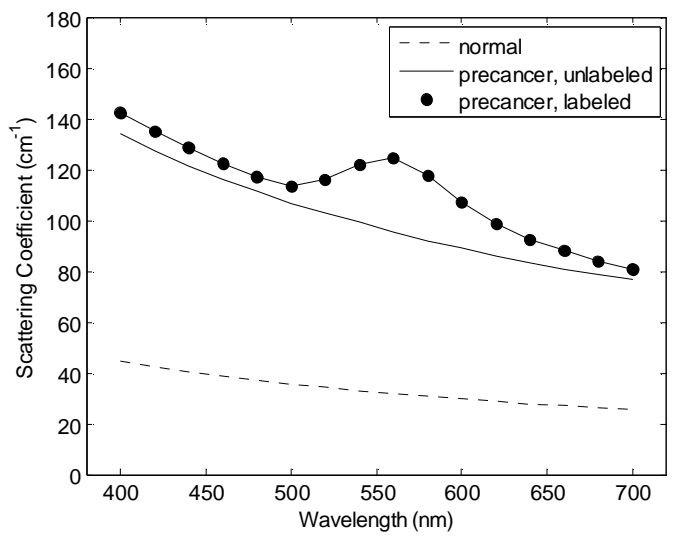

(a)

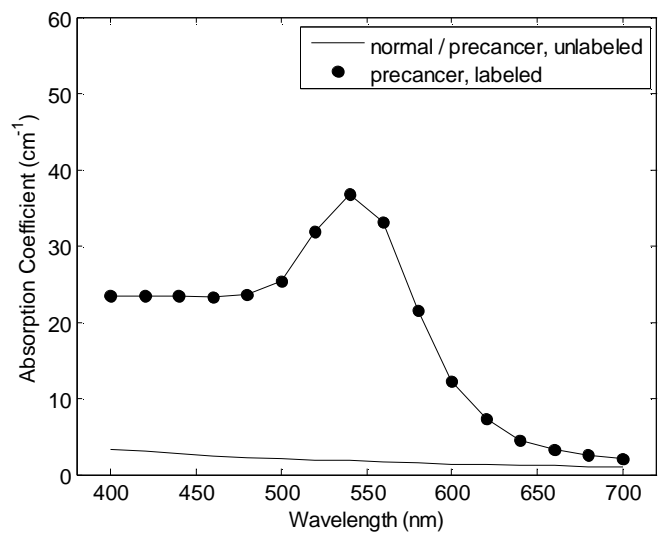

(b)

Figure 1. (a) Scattering and (b) absorption coefficients of normal epithelium, unlabeled precancerous epithelium, and precancerous epithelium labeled with 80 -nm gold nanospheres (volume fraction $=0.005 \%$ ). 
Sample angular scattering probability distributions for the epithelial layer are shown in Fig. 2 for four different wavelengths. Since we assume that scattering properties of normal and unlabeled precancerous epithelium are characterized by Henyey-Greenstein phase functions with a fixed anisotropy factor of 0.95 , the probability distributions for these two tissue states have the same values. Note that functions denoted as $p(\theta)$ have been normalized such that the sum of scattering probabilities for $\theta=\{0,1, \ldots, 180\}$ equals one in each case.

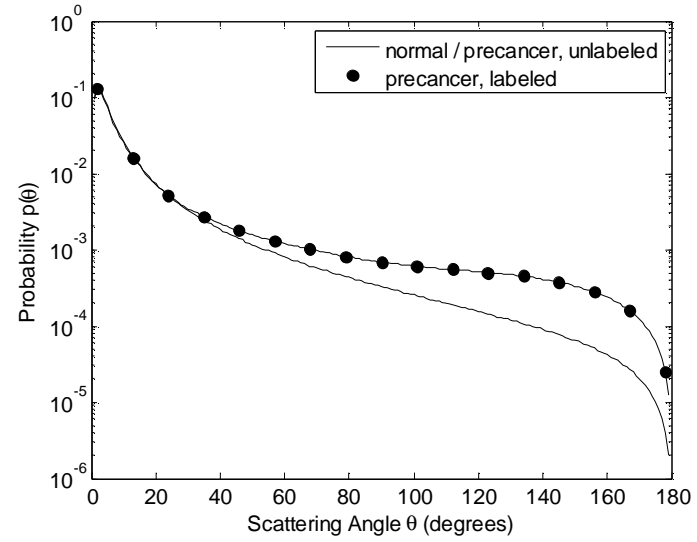

(a)

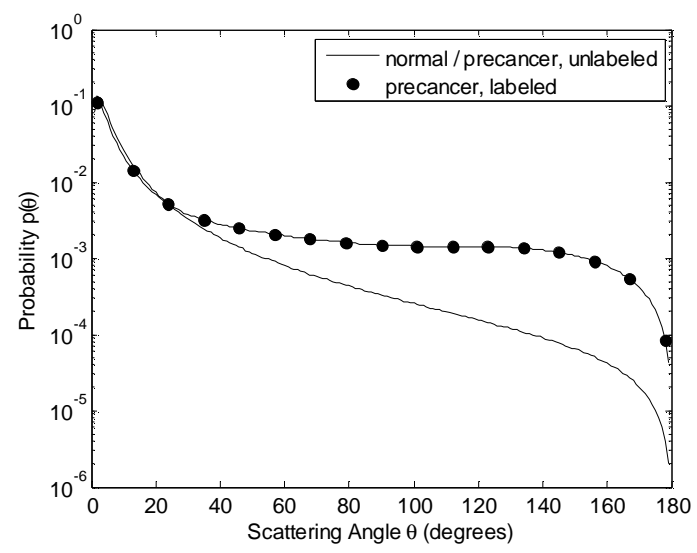

(b)

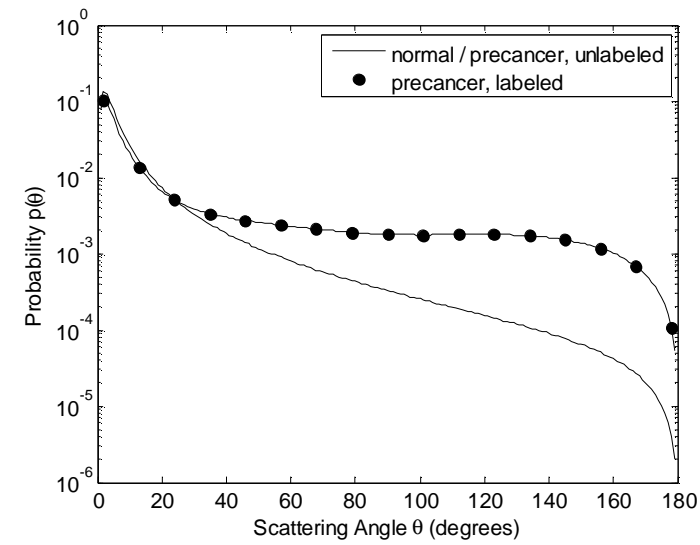

(c)

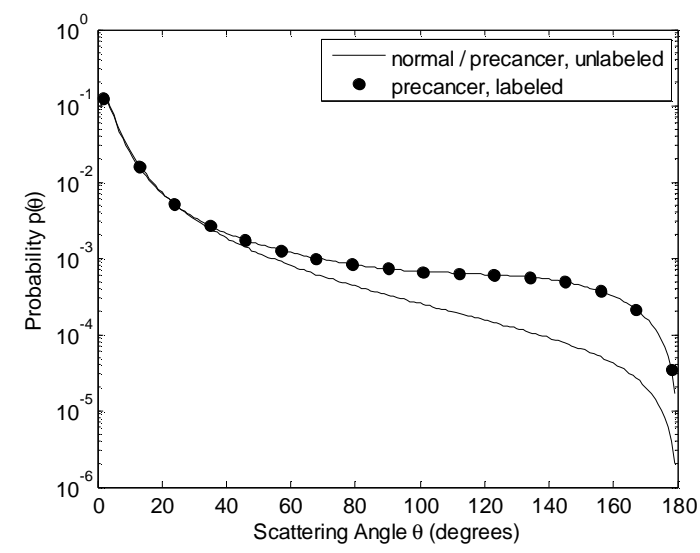

(d)

Figure 2. Sample phase functions for normal epithelium, unlabeled precancerous epithelium, and precancerous epithelium labeled with $80-\mathrm{nm}$ gold nanospheres (volume fraction=0.005\%). Four different wavelengths are shown: (a) $420 \mathrm{~nm}$, (b) $540 \mathrm{~nm}$, (c) $560 \mathrm{~nm}$, and (d) $680 \mathrm{~nm}$; the anisotropy factors for labeled epithelium are 0.89 , $0.78,0.74$, and 0.90 , respectively.

Figures 1 and 2 demonstrate that addition of nanospheres can significantly change the optical properties of the epithelial layer. Increased high-angle $\left(>\sim 20^{\circ}\right)$ scattering probability is also evident for labeled epithelium.

\subsection{Detected reflectance intensity}

Figure 3 shows the spectral reflectance profile of different tissue states when the source and detector fibers are oriented perpendicular to the tissue surface [Fig. 3(a)] or when the fibers are tilted at $30^{\circ}$ with respect to the tissue normal [Fig. 3(b)]. Also shown in Fig. 3 are the error bars corresponding to the standard error values over three simulations. 


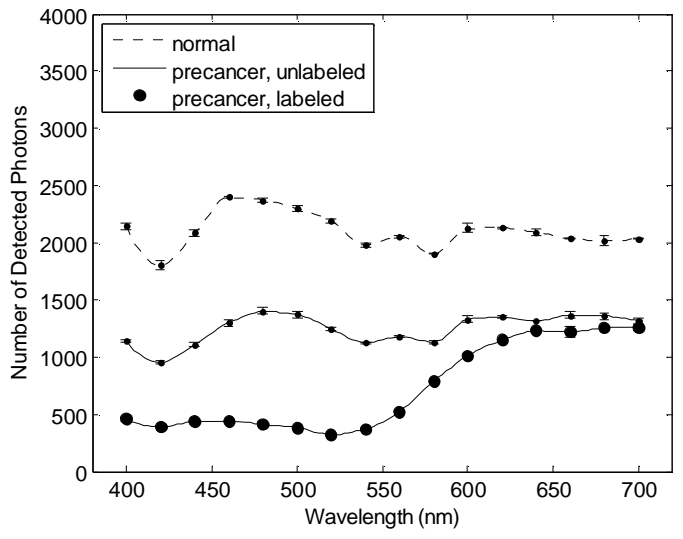

(a)

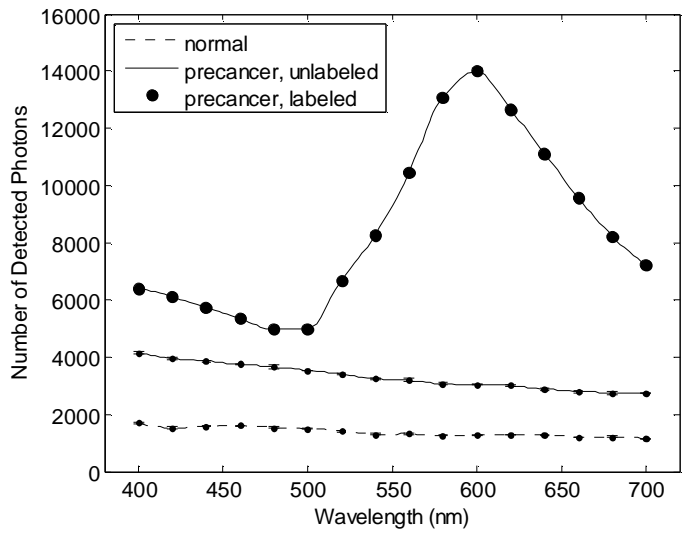

(b)

Figure 3. Spectral reflectance profile of normal tissue, unlabeled precancerous tissue, and precancerous tissue labeled with $80-\mathrm{nm}$ gold nanospheres (volume fraction=0.005\%). In (a), the source and detector fibers are perpendicular to the tissue surface and in (b), the fibers are tilted toward each other.

The results in Fig. 3 indicate that addition of gold nanospheres can lead to a negative or a positive contrast enhancement depending on the particular source-detector geometry used. For perpendicular fibers, the reflectance intensity of precancerous tissue is lower compared to that of normal tissue over the entire wavelength range; addition of nanospheres enhances this negative contrast, especially for wavelengths smaller than $600 \mathrm{~nm}$. For tilted fibers, on the other hand, the reflectance profile of precancerous tissue is characterized by a positive contrast relative to that of normal tissue; labeling with nanospheres causes a significant increase in reflectance intensity and the extent of this contrast enhancement is maximum at $\sim 600 \mathrm{~nm}$.

\subsection{Penetration depth profiles}

It is also important to monitor the penetration depths of detected photons in order to assess the effect of labeling on photon propagation characteristics. In Fig. 4, the maximum penetration depths averaged over all detected photons are plotted for perpendicular and tilted fibers [Figs. 4(a) and 4(b), respectively]. The error bars representing the standard error values over three simulations are also shown.

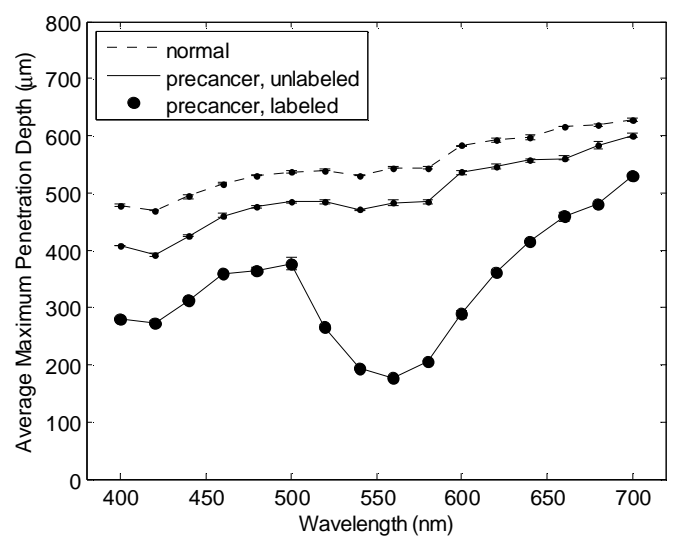

(a)

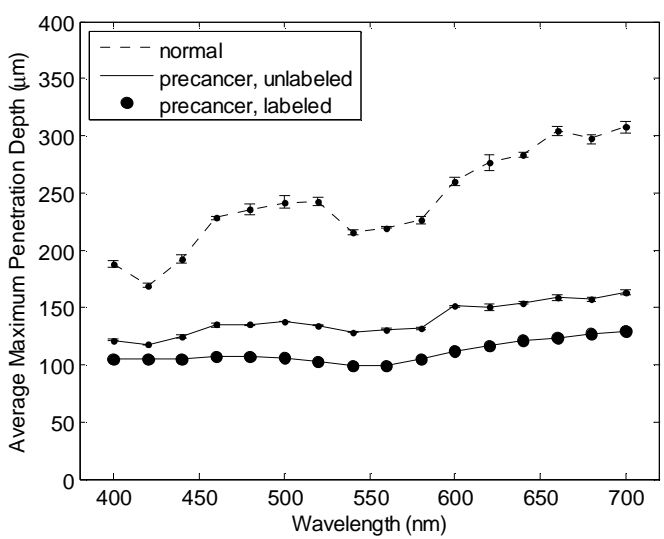

(b)

Figure 4. Average maximum penetration depths of detected photons for normal tissue, unlabeled precancerous tissue, and precancerous tissue labeled with 80 -nm gold nanospheres (volume fraction $=0.005 \%$ ). In (a), the source and detector fibers are perpendicular to the tissue surface and in (b), the fibers are tilted toward each other.

The results presented in Fig. 4 suggest that addition of gold nanospheres leads to a decrease in average maximum penetration depth of detected photons over the entire spectral range. For perpendicular fibers, the decrease in average maximum penetration depth is most prominent for wavelengths between 520 and $620 \mathrm{~nm}$; photons appear to propagate 
mostly through the epithelial layer without entering the stroma. Tilted fibers are preferentially sensitive to epithelial optical signals for both normal and precancerous tissue; labeling with nanospheres confines photons to more superficial depths within the epithelium.

\subsection{Layer selectivity}

Figure 5 shows the percentage of detected photons that only propagate through the top epithelial layer without penetrating into the stroma. The error bars again represent the standard error values computed over three simulations.

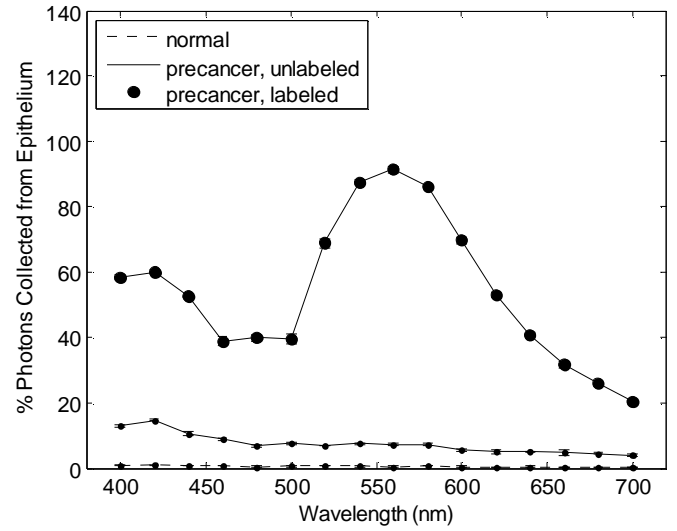

(a)

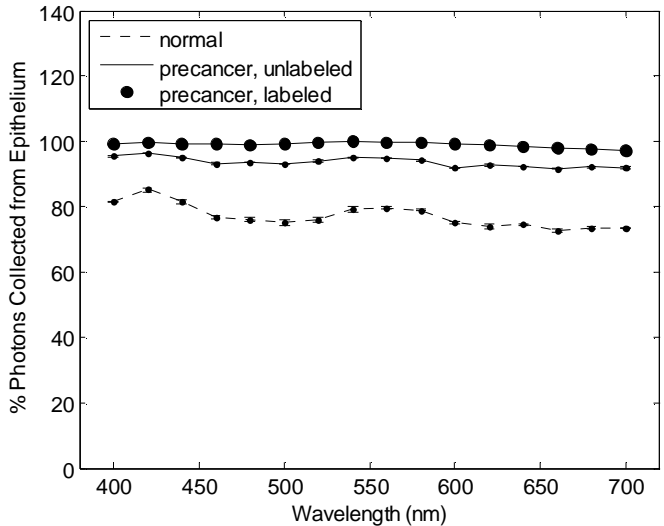

(b)

Figure 5. Percentage of photons collected from epithelium for normal tissue, unlabeled precancerous tissue, and precancerous tissue labeled with $80-\mathrm{nm}$ gold nanospheres (volume fraction $=0.005 \%$ ). In (a), the source and detector fibers are perpendicular to the tissue surface and in (b), the fibers are tilted toward each other.

The percentage values plotted in Fig. 5 corroborate the results displayed in Fig. 4. When the fibers are oriented perpendicular to the surface, the majority of photons collected from unlabeled tissue penetrate into the stromal layer and optical signals do not retain information on epithelial properties. Addition of nanospheres can lead to significant changes in layer selectivity, but this appears to be highly dependent on wavelength; the percentage of photons collected from the epithelial layer increases to $\sim 90 \%$ at $560 \mathrm{~nm}$. Tilted fibers exhibit a high degree of selectivity for epithelial optical signals even for unlabeled tissue. When precancerous tissue is labeled with nanospheres, epithelial selectivity reaches almost $100 \%$ for all the wavelengths simulated.

\section{DISCUSSION AND CONCLUSIONS}

Model-based analysis of photon propagation through nanoparticle-labeled tissues is expected to provide a quantitative understanding of the extent of contrast enhancement due to external labeling. The results obtained in this study indicate that nanoparticle-induced changes in the reflectance response of tissues depend not only on the properties of these particles but also on the source-detector geometry used. When the fibers are oriented perpendicular to the tissue surface, addition of nanospheres leads to a decrease in reflectance intensity of precancerous tissue. This suggests that nanoparticle-induced changes in epithelial absorption properties dominate and cause an enhancement of the inherent negative contrast relative to normal tissue. When the fibers are tilted, however, nanoparticle-induced changes in epithelial scattering properties dominate leading to an increase in reflectance intensity and an enhancement of the inherent positive contrast relative to normal tissue. These observations suggest that a comprehensive investigation is needed to meaningfully interpret detected optical signals and to assess the diagnostic potential of nanoparticle-enhanced optical measurements. 


\section{REFERENCES}

[1] Brown, J. Q., Vishwanath, K., Palmer, G. M. and Ramanujam, N., "Advances in quantitative UV-visible spectroscopy for clinical and pre-clinical application in cancer," Curr. Opin. Biotechnol. 20, 119-131 (2009).

[2] Sokolov, K., Follen, M., Aaron, J., Pavlova, I., Malpica, A., Lotan, R. and Richards-Kortum, R., "Real-time vital optical imaging of precancer using anti-epidermal growth factor receptor antibodies conjugated to gold nanoparticles," Cancer Res. 63, 1999-2004 (2003).

[3] Hirsch, L. R., Gobin, A. M., Lowery, A. R., Tam, F., Drezek, R. A., Halas, N. J. and West, J. L., "Metal nanoshells," Annals Biomed. Eng. 34, 15-22 (2006).

[4] Wax, A. and Sokolov, K., "Molecular imaging and darkfield microspectroscopy of live cells using gold plasmonic nanoparticles," Laser \& Photon. Rev. 3, 146-158 (2009).

[5] Lin, A. W. H., Lewinski, N. A., West, J. L., Halas, N. J. and Drezek, R. A., "Optically tunable nanoparticle contrast agents for early cancer detection: model-based analysis of gold nanoshells," J. Biomed. Opt. 10, 064035 (2005).

[6] Kirillin, M., Shirmanova, M., Sirotkina, M., Bugrova, M., Khlebtsov, B. and Zagaynova, E., "Contrasting properties of gold nanoshells and titanium dioxide nanoparticles for optical coherence tomography imaging of skin: Monte Carlo simulation and in vivo study," J. Biomed. Opt. 14, 021017 (2009).

[7] Arifler, D., Schwarz, R. A., Chang, S. K. and Richards-Kortum, R., "Reflectance spectroscopy for diagnosis of epithelial precancer: model-based analysis of fiber-optic probe designs to resolve spectral information from epithelium and stroma," Appl. Opt. 44, 4291-4305 (2005).

[8] Arifler, D., MacAulay, C., Follen, M. and Richards-Kortum, R., "Spatially resolved reflectance spectroscopy for diagnosis of cervical precancer: Monte Carlo modeling and comparison to clinical measurements," J. Biomed. Opt. 11, 064027 (2006).

[9] Kortun, C., Hijazi, Y. R. and Arifler, D., "Combined Monte Carlo and finite-difference time-domain modeling for biophotonic analysis: implications on reflectance-based diagnosis of epithelial precancer," J. Biomed. Opt. 13, 034014 (2008).

[10] Mourant, J. R., Freyer, J. P., Hielscher, A. H., Eick, A. A., Shen, D. and Johnson, T. M., "Mechanisms of light scattering from biological cells relevant to noninvasive optical-tissue diagnostics," Appl. Opt. 37, 3586-3593 (1998). 